\title{
hot off the press
}

\section{Wnts need a p(assport)24 to leave the ER}

\author{
Lucy Palmer, Jean-Paul Vincent \& Karen Beckett
}

W nt signalling proteins regulate diverse cellular processes during development and homeostasis. Since misregulation of Wnt signalling is associated with cancer, most research has been aimed at characterizing the signal transduction pathway. Recently, attention has focused on Wnt production due to the identification of factors required specifically for Wnt secretion. For instance, the specific requirement of Evi, also known as Wntless or Sprinter, suggested that Wnts might follow a specialized secretory route (Banziger et al, 2006; Bartscherer et al, 2006). Two recent papers published in EMBO reports by the groups of Konrad Basler in last month's issue, and Michael Boutros in this issue, show that Wnt secretion requires the activity of p24 family members (Buechling et al, 2011; Port et al, 2011). Therefore, the specialized route might begin at endoplasmic reticulum (ER) exit sites.

Wnts are secreted glycoproteins that can act many cell diameters from their source of production. Most, but not all Wnt proteins, are acylated and thus associate tightly with cellular membranes. Despite this association, acylated Wnts can be released from secreting cells and spread in the extracellular space (Bartscherer \& Boutros, 2008; Port \& Basler, 2010). Acylation of Wnts, which occurs in the ER, is thought to be mediated by the $\mathrm{N}$-acetyl transferase encoded by porcupine (porc; van den Heuvel et al, 1993). After acylation, Wnt proteins associate with Evi, a multipass transmembrane protein found mostly at the Golgi and the plasma membrane (Fig 1A). This association is essential for the secretion of the Wnts that are acylated since, in the absence of Evi, they accumulate on internal membranes (Banziger et al, 2006; Bartscherer et al, 2006). It is therefore thought that acylated Wnts require Evi to exit the Golgi and progress to the cell surface (Port et al, 2008).
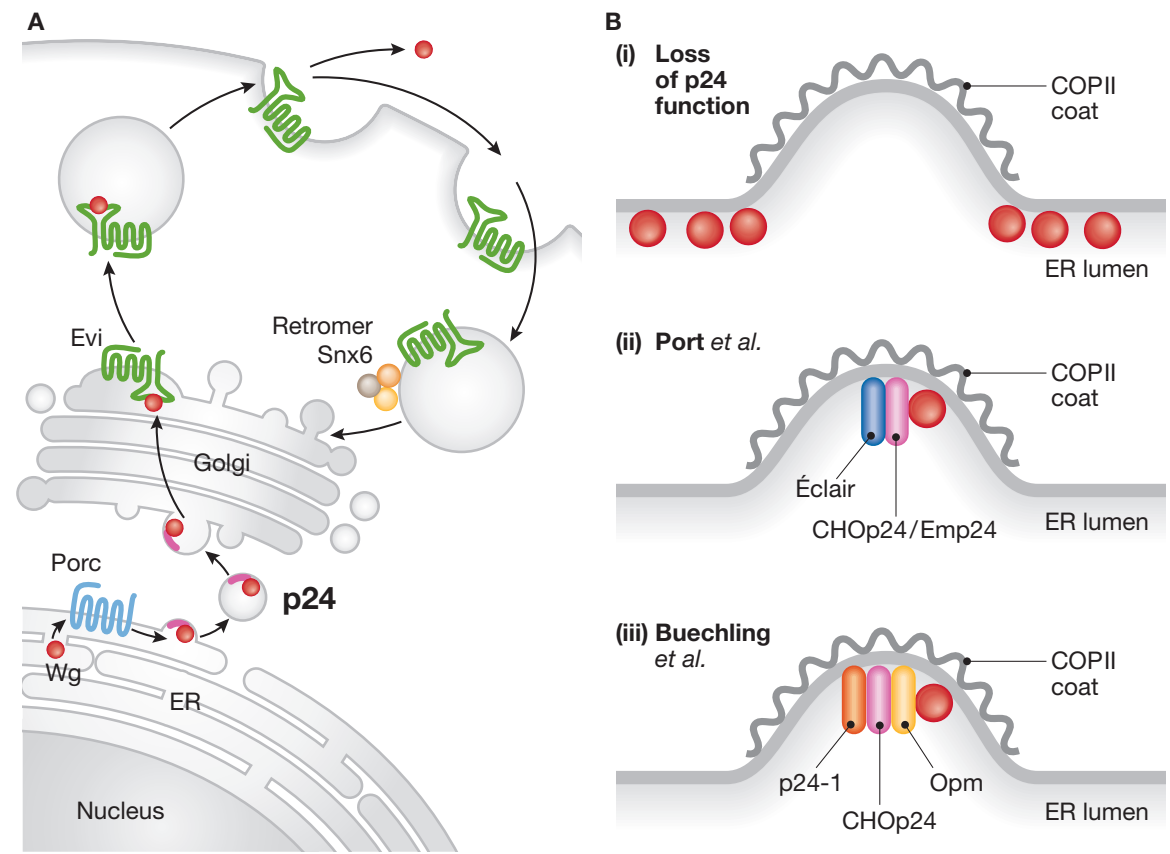

Fig 1 | Model summarizing the suggested roles of p24 proteins in endoplasmic reticulum to Golgi transport of Wg. (A) Schematic of a cell showing the current model of the Wg secretory pathway. Wg is produced in the ER where it is lipid-modified by Porc, and moved to the Golgi with the assistance of p24 proteins. In the Golgi, Wg joins Evi, which facilitates Wg transport to the cell surface. Evi is then recycled back to the Golgi in a path mediated by the retromer complex. (B)(i) In the absence of p24 proteins, there is no recruitment of Wg to COPII-coated vesicles and therefore a block of secretion in the ER. (ii) Port et al (2011) propose a similar model in which CHOp24/Emp24 and Éclair are involved in Wg recruitment, although only CHOp24/ Emp24 binds to Wg. (iii) According to Buechling et al (2011), Opm recruits Wg to COPII-coated vesicles for movement to the Golgi. CHOp24 and p24-1 are also required for this process. ER, endoplasmic reticulum.

This does not seem to be a requirement for non-lipidated Wnts, such as Drosophila WntD, which are secreted in the absence of Evi (Ching et al, 2008). Similarly, secretion of other signalling proteins proceeds normally without Evi. After reaching the cell surface, Evi is likely to have a choice between various routes. One such route, which involves the retromer complex, takes it back to the Golgi where it can participate in another round of Wnt secretion (Fig 1A). Alternatively, Evi can be targeted to lysosomes (Bartscherer \& Boutros, 2008;
Port \& Basler, 2010). The factors that determine Evi transport remain poorly understood. Nevertheless, these studies highlight the essential and specific role of Evi for the secretion of lipidated Wnts.

Two groups have now reported the use of cell-based RNA interference (RNAi) screens to identify further proteins required for the secretion of Wingless $(\mathrm{Wg})$, the main Drosophila Wnt (Buechling et al, 2011; Port et al, 2011). They found that p24 family members, a group of proteins previously implicated in both retrograde 
and anterograde transport between the ER and Golgi (Strating \& Martens, 2009), are required for $\mathrm{Wg}$ secretion by $\mathrm{S} 2$ cells. Similarly, knockdown by transgenic RNAi shows that p24 proteins are required for normal levels of Wg secretion in Drosophila wing imaginal discs (Buechling et al, 2011; Port et al, 2011). As with Evi, this requirement seems to be relatively specific, since general secretion and the secretion of other signalling proteins, including the lipid-modified morphogen Hedgehog, are unaffected by p24 knockdown. Buechling et al also assessed the role of p24 proteins in WntD secretion. They found that RNAi against opossum (opm), one of the p24 members, prevents WntD secretion in cultured cells. They also show that the phenotypes of opm mutants and WntD mutant embryos resemble each other (Buechling et al, 2011). Therefore, while Evi is specifically required for the secretion of acylated Wnts, p24 proteins could contribute to the secretion of all Wnts. This function is likely to be conserved since the mammalian homologue of Opm, TMED5, is required for Wnt1 signalling, at least in a mammalian cell culture assay (Buechling et al, 2011).

To gain understanding of the role of p24 proteins in Wnt secretion, both groups analysed the subcellular localization of Wg following p24 knockdown. They found accumulation in the ER and concomitant depletion in the Golgi, as indicated by reduced co-localization with Golgi markers (Fig 1Bi). They also found that p24 knockdown prevents $\mathrm{Wg}$ from stabilizing Evi in producing cells, suggesting that the stabilizing influence of $\mathrm{Wg}$ requires its exit from the ER (Buechling et al, 2011; Port et al, 2011). These results lead the authors to propose that the loss of p24 prevents the transport of Wg from the ER to the Golgi. Importantly, immunoprecipitation experiments suggest that Wg might interact physically with Opm and Emp24 (also known as CHOp24). This led both sets of authors to postulate a model whereby p24 proteins act as cargo receptors to escort Wnt proteins from the ER to the Golgi, whereupon they can bind to Evi, which will escort them to the plasma membrane. Thus, in this context, p24 proteins seem to have an anterograde function.

Although both studies highlight the role of p24 proteins in Wnt secretion, they disagree on the relative importance of the various family members. Among the nine predicted p24 proteins encoded by the Drosophila genome, only Éclair and Emp24/CHOp24 were found to be required for $\mathrm{Wg}$ secretion by Port et al (2011; Fig 1Bii). By contrast, Buechling et al found that Opm, Emp24/CHOp24 and p24-1 all play a role in $\mathrm{Wg}$ secretion (fig 1Biii). Thus, only Emp24/CHOp24 is found by both groups to be essential for $\mathrm{Wg}$ secretion. Although functional redundancy among p24 proteins could explain why the removal of a single $\mathrm{p} 24$ protein has a relatively weak phenotype, there is no simple explanation as to why the very similar assays used by the two groups do not lead to identical conclusions. These differences could be worked out by the exchange of reagents and protocols.

Regardless of the discrepancies, the two studies provide an important step in our understanding of Wnt secretion by demonstrating that Wnts engage with specialized components of the secretory machinery as early as in the ER. It might be relevant that the anterograde function of p24 proteins is directed at glycophosphatidylinositol (GPI)-anchored proteins, which have been shown to partition in raft-like microdomains (Strating \& Martens, 2009). It is conceivable that GPI-anchored proteins, as well as Wnts, gather in a subdomain of the ER where they could both interact with p24 proteins and set off along their specialized secretory pathways. Wnt targeting to specialized membrane domains could in principle be mediated by their lipid moieties (Bartscherer \& Boutros, 2008; Port \& Basler, 2010). However, the process might turn out to be more complex if it is confirmed that p24 proteins are also required for the secretion of non-acylated Wnts (for example, WntD), as suggested by Buechling et al. In any case, it will be interesting to determine the precise molecular mechanism underlying the functional interaction between Wnts and p24 proteins as it is likely to explain how Wnts are allowed to exit the ER and start their journey out of the cell.

\section{REFERENCES}

Banziger C et al (2006) Cell 125: 509-522

Bartscherer K, Boutros M (2008) EMBO Rep 9: 977-982

Bartscherer K et al (2006) Cell 125: 523-533

Buechling T et al (2011) EMBO Rep 12: 1265-1272

Ching W et al (2008) J Biol Chem 283: 17092-17098

Port F, Basler K (2010) Traffic 11: 1265-1271

Port F et al (2008) Nat Cell Biol 10: 178-185

Port F et al (2011) EMBO Rep 12: 1144-1152

Strating JR, Martens GJ (2009) Biol Cell 101: 495-509

van den Heuvel M et al (1993) EMBO / 12:

5293-5302

Lucy Palmer, Jean-Paul Vincent and Karen

Beckett are at the MRC National Institute

for Medical Research, Mill Hill, London

NW7 1AA, UK.

E-mail:kbecket@nimr.mrc.ac.uk

Published online 18 November 2011

EMBO reports (2011) 12, 1207-1208.

doi:10.1038/embor.2011.222

\section{Pol II caught speeding by single gene imaging Danielle Cannon \& Jonathan R. Chubb}

T he transcription elongation rate of RNA polymerase II (Pol II) has been re-estimated persistently for more than 30 years using a variety of methods. A new imaging-based estimate of elongation rate on an HIV-derived transgene implies Pol II can elongate at least an order-of-magnitude faster than previously thought.

From early pulse-labelling experiments to recent studies monitoring post-stimulus waves of transcription using Pol II chromatin immunoprecipitation (ChIP), tiling arrays or reverse transcription (RT)-PCR, estimates of $\mathrm{Pol}$ II elongation rate from various eukaryotic cell types have ranged between 1 and $6 \mathrm{~kb} / \mathrm{min}$ (Ardehali \& Lis, 2009; Wada et al, 2009). Imaging approaches, using differences in hybridization of differently positioned RNA fluorescence in situ hybridization (FISH) probes, and more recently live-cell RNA detection, have generated elongation rates similar to those measured using test-tube processing methods. Quite unexpectedly, a study by Marcello and colleagues published in this issue of EMBO reports shows that the RNA Pol II elongation rate can be as fast as $100 \mathrm{~kb} / \mathrm{min}$ (Maiuri et al, 2011). 